\title{
Méthodologie de l'Enquête sur les personnes ayant une maladie chronique au Canada - composante de l'hypertension de 2009
}

\author{
A. S. Bienek, M.G.S.S. (1); M. E. Gee, M. Sc. (1); R. P. Nolan, Ph. D. (2); J. Kaczorowski, Ph. D. (3); N. R. Campbell, M.D. (4); \\ C. Bancej, Ph. D. (1); F. Gwadry-Sridhar, Ph. D. (5); C. Robitaille, M. Sc. (1); R. L. Walker, M. Sc. (6); S. Dai, M.D. (1)
}

Cet article a fait l'objet d'une évaluation par les pairs.

\section{Résumé}

Introduction : L’Enquête sur les personnes ayant une maladie chronique au Canada composante de l'hypertension (EPMCC-H) est une enquête téléphonique transversale de 20 minutes sur le diagnostic et la prise en charge de l'hypertension. L'échantillon de l'EPMCC-H, sélectionné à partir des répondants à l'Enquête sur la santé dans les collectivités canadiennes (ESCC) de 2008, était composé de Canadiens (de 20 ans et plus) des dix provinces ayant déclaré avoir reçu un diagnostic d'hypertension.

Méthodologie : Le questionnaire a été élaboré au moyen de la technique Delphi et a fait l'objet d'un examen externe ainsi que de tests qualitatifs. Statistique Canada s'est chargé des stratégies d'échantillonnage, du recrutement, de la collecte et du traitement des données. Les proportions ont été pondérées afin de représenter la population canadienne et les intervalles de confiance (IC) à $95 \%$ ont été calculés au moyen de la méthode de rééchantillonnage bootstrap.

Résultats : Si on le compare à la population de l'ESCC ayant déclaré souffrir d'hypertension, l'échantillon de l'EPMCC-H ( $\mathrm{n}=6$ 142) est légèrement plus jeune (âge moyen des répondants à l'EPMCC-H : 61,2 ans, IC à $95 \%$ : 60,8 à 61,6; âge moyen des répondants à l'ESCC : 62,2 ans, IC à $95 \%: 61,8$ à 62,5), comporte plus de détenteurs d'un diplôme d'études postsecondaires (EPMCC-H : 52,0 \%, IC à $95 \%$ : 49,7 \% à 54,2 \%; ESCC : 47,5\%, IC à $95 \%: 46,1 \%$ à 48,9\%) et moins de répondants prenant un médicament pour l'hypertension (EPMCC-H : 82,5\%, IC à $95 \%: 80,9 \%$ à 84,1\%; ESCC : $88,6 \%$, IC à $95 \%: 87,7 \%$ à 89,6\%).

Conclusion : Dans l'ensemble, l'EPMCC-H de 2009 est représentatif de sa population source et fournit des données nouvelles et exhaustives sur le diagnostic et la prise en charge de l'hypertension. L'enquête a été adaptée à d'autres maladies chroniques diabète, asthme/maladie pulmonaire obstructive chronique et troubles neurologiques. Le questionnaire est accessible à partir du site Web de Statistique Canada; des résultats descriptifs ont été publiés par l'Agence de la santé publique du Canada.

Mots-clés : enquête épidémiologique, hypertension, maladie chronique, collecte de données, enquêtes sur la santé, questionnaires, Enquête sur la santé dans les collectivités canadiennes

\section{Introduction}

Plus du cinquième des Canadiens âgés de plus 20 ans ont reçu un diagnostic d'hypertension $^{1,2}$, et $17 \%$ de la population adulte en souffre probablement sans le savoir ${ }^{3}$. La pression artérielle élevée est un facteur étiologique important du risque de maladie cardiovasculaire, mais il est possible de la contrôler efficacement en modifiant son mode de vie, c'est-à-dire son niveau d'activité physique, son alimentation, son apport en sodium, sa consommation d'alcool et sa consommation de tabac, en contrôlant son poids, ou grâce à la pharmacothérapie, au besoin ${ }^{4}$. Malgré cela, environ $33 \%$ des Canadiens ayant reçu un diagnostic d'hypertension ont une pression artérielle élevée qui n'est pas bien maîtrisée $e^{3}$. Une meilleure compréhension des connaissances, de l'attitude et des comportements des Canadiens ayant reçu un diagnostic d'hypertension aiderait à l'élaboration et à l'amélioration de programmes axés sur le contrôle de la pression artérielle.

En 2009, l'Agence de la santé publique du Canada (ASPC) a mené l'Enquête sur les personnes ayant une maladie chronique au Canada - composante de l'hypertension (EPMCC-H) afin de déterminer comment les Canadiens vivent avec leur hypertension et la prennent en charge. Cette enquête de 20 minutes, la première à

Rattachement des auteurs :

1. Agence de la santé publique du Canada, Ottawa (Ontario), Canada

2. University Health Network, Toronto (Ontario), Canada

3. Département de médecine familiale et médecine d'urgence, Université de Montréal, Centre de recherche du CHUM, Hôpital Notre-Dame, Montréal (Québec), Canada

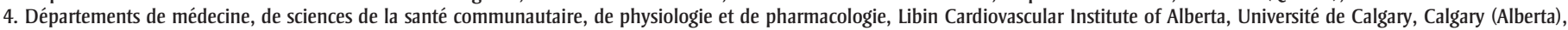
Canada

5. Institut de recherche en santé Lawson, Université Western Ontario, London (Ontario), Canada

6. Département des sciences de la santé communautaire, Université de Calgary, Calgary (Alberta), Canada

Correspondance : Asako S. Bienek, Agence de la santé publique du Canada, 785, avenue Carling, IA : 6806A, Ottawa (Ontario) K1A 0K9; tél. : 613-952-6163; téléc. : 613-941-2057; courriel : Asako.Bienek@phac-aspc.gc.ca 
être menée auprès d'un échantillon représentatif à l'échelle nationale des Canadiens chez qui une maladie chronique a été diagnostiquée, fournit de nouvelles variables utilisables pour la surveillance d'indicateurs relatifs à la santé et pour la production de rapports à ce sujet. Cet article décrit les objectifs et la méthodologie de l'EPMCC-H de 2009 et analyse la représentativité de l'échantillon final.

\section{Méthodologie}

\section{Objectifs de l'enquête}

L'ASPC a entrepris l'EPMCC en 2006 pour évaluer l'impact des maladies chroniques sur la qualité de vie des personnes et de leurs familles, pour recueillir des renseignements sur la façon dont les personnes prennent en charge leurs maladies chroniques, pour déterminer l'utilité des interventions relatives à la prise en charge des maladies chroniques chez les membres de la collectivité, pour cerner les comportements en matière de santé qui influent sur les effets de la maladie et pour analyser les obstacles à l'autogestion des maladies chroniques. L'ASPC a sélectionné l'hypertension et l'arthrite pour la première version de l'EPMCC, après avoir pris en considération l'importance de ces maladies du point de vue de la santé publique, l'existence de travaux de surveillance complémentaires à l'échelle nationale, ainsi que la prévalence et la taille de l'échantillon de personnes souffrant de plusieurs maladies chroniques. Après avoir consulté Statistique Canada, l'ASPC a déterminé que cette enquête ne nécessitait aucune approbation sur le plan éthique étant donné qu'aucune mesure physique ne serait entreprise. Aucun risque pour les renseignements personnels ou la confidentialité, qui sont régis par la Politique d'évaluation des facteurs relatifs à la vie privée, n'a été cerné, et le statisticien en chef de Statistique Canada a autorisé le lancement de l'enquête.

\section{Élaboration du contenu de l'enquête}

En 2007, l'ASPC a collaboré avec le Programme éducatif canadien sur l'hypertension $(\mathrm{PECH})$ afin de mettre sur pied un groupe de travail dont les membres étaient dotés d'une expertise en matière d'hypertension ou d'élaboration et de validation d'enquêtes. Le groupe de travail a élaboré le questionnaire administré par téléphone utilisé dans le cadre de cette enquête transversale. Les questions ont été tirées d'enquêtes démographiques publiées, notamment du contenu obligatoire, thématique et facultatif des divers cycles de l'Enquête sur la santé dans les collectivités canadiennes $(\mathrm{ESCC})^{5}$; du cycle 4 de l'Enquête nationale sur la santé de la population $(\mathrm{ENSP})^{6}$; des questionnaires relatifs à la pression artérielle et aux maladies cardiovasculaires de la National Health and Nutrition Examination Survey des États-Unis (NHANES) de 2005-20067 ; de l'enquête Harris Interactive Hypertension Education menée aux ÉtatsUnis (2007) ${ }^{8}$ ainsi que d'enquêtes portant sur des thèmes précis tels que l'activité physique ou l'alimentation. Des articles soumis à une évaluation par les pairs ont été consultés afin de trouver d'autres instruments de mesure et des échelles reconnues, par exemple des échelles d'auto-efficacité générale ou l'échelle Morisky sur l'observance de médicaments pour l'hypertension ${ }^{9-11}$. Certaines questions relatives à la prise en charge et à la surveillance de la pression artérielle ont été adaptées, à la suite de consultations avec des experts et d'après les lignes directrices nationales existantes, en particulier celles publiées par le $\mathrm{PECH}^{12,13}$, les National Institutes of Health $^{14}$ et le National Cholesterol Education Program des États-Unis ${ }^{15}$.

Un examen préliminaire a permis de déterminer si les questions étaient adaptées à l'âge et à la population, si elles pouvaient être posées au téléphone et si elles correspondaient à la portée de l'EPMCC tout en étant suffisamment générales pour pouvoir être adaptées à d'autres maladies chroniques et utilisées dans des versions ultérieures de l'enquête. En se servant de l'ESCC comme guide, le groupe de travail a organisé les questions conservées par thème et les a reformulées en se concentrant sur leur ordre et leur enchaînement, leur normalisation, les catégories et les plages de réponse, ainsi que sur l'uniformisation du niveau de langue et l'utilisation de la forme narra- tive. Le biais lié aux réponses a été pris en considération en supprimant certaines questions suggestives ou répétitives. Les contraintes de temps associées à une entrevue téléphonique et la fatigue des répondants ont également dicté la longueur de l'enquête.

L’approche Delphi a été utilisée pour atteindre un consensus quant au contenu. En général, les questions dont les réponses seraient difficiles à analyser ou à interpréter ont été supprimées. De même, celles dont les réponses seraient difficiles à transposer en recommandations pratiques ont également été supprimées. Ont été écartés les concepts qui étaient déjà ciblés dans l'ESCC principale et sur lesquels des renseignements pouvaient donc être obtenus par jumelage (p. ex. nutrition ou activité physique), qui étaient trop vastes pour être traités adéquatement (p. ex. indice de l'état de santé, étapes du changement), qui nécessitaient une explication détaillée (p. ex. attentes relatives à l'auto-efficacité) ou qui auraient mené à des catégories de réponses trop peu nombreuses pour être analysées. Le contenu final de la version anglaise de l'enquête a été traduit en français pour pouvoir mettre en œuvre l'enquête dans les deux langues officielles du Canada et l'exactitude du contenu traduit a été vérifiée.

\section{Examen externe}

À l'aide d'une version provisoire du questionnaire, 15 membres du PECH (taux de réponse de $30 \%$ ) ont examiné l'enquête et donné une rétroaction détaillée, ce qui a permis de confirmer le choix des principales catégories de contenu et de combler les éventuelles lacunes. Certaines des recommandations formulées excédaient la portée de l'enquête, par exemple le rappel alimentaire de 24 heures, le recours à des cliniques spécialisées, la surveillance ambulatoire de la pression artérielle, l'exploration d'autres maladies macrovasculaires et le risque global de maladie cardiovasculaire. Cependant, des catégories ont été ajoutées ou élargies, notamment l'utilité et la disponibilité de matériel éducatif écrit sur l'hypertension, la connaissance des principaux enjeux et 
les obstacles à l'observance des modifications apportées au mode de vie.

\section{Tests qualitatifs}

Statistique Canada a soumis les versions anglaise et française de l'enquête à des tests qualitatifs afin d'en évaluer la clarté, la validité apparente, le déroulement du questionnaire, la facilité d'administration ainsi que les réponses auprès d'un souséchantillon de répondants souffrant d'hypertension ou ayant déjà reçu un diagnostic d'hypertension (quel que soit leur traitement pharmacologique contre l'hypertension), sélectionnés au hasard parmi environ $10 \quad 000$ répondants à l'ESCC de 2007. Tout a été mis en œuvre pour obtenir un échantillon le plus diversifié possible en termes d'âge, de sexe, de niveau de scolarité, de revenu et de lieu de résidence (centre-ville/grande région métropolitaine). Le consentement verbal a été obtenu au moment de la sélection des participants, qui ont été informés que l'entrevue serait enregistrée et se déroulerait sous l'observation de membres du personnel.

Une heure a été accordée pour chaque entrevue en personne. Sur les 16 entrevues prévues, 13 ont été menées à terme (8 en anglais et 5 en français). Pendant les entrevues, des membres de l'équipe observaient les réactions des participants à propos du contenu ainsi que leur volonté et leur capacité de fournir des réponses. Les intervieweurs ont posé des questions aux participants sur les mesures de leur pression artérielle et sur l'observance de leurs médicaments pour l'hypertension et leur ont demandé de formuler des commentaires généraux à propos du contenu de l'enquête. En raison de la petite taille de l'échantillon, les résultats ont été utilisés pour leur valeur qualitative et n’ont pas été considérés comme représentatifs du point de vue statistique. Au moment des tests, il a fallu entre 30 et 40 minutes pour l'administration du questionnaire, d'où la nécessité de le raccourcir afin de réduire de 15 minutes la durée totale de l'entrevue (compte tenu du fait que la version française était plus longue). De plus, l'ordre des questions a été revu afin d'améliorer le déroulement de l'entrevue, les questions délicates et les périodes de référence ont été modifiées, le niveau de langue a été simplifié, la terminologie et les traductions ont été clarifiées et les clés de correction et les questions à sauter ont été revues afin de mieux tenir compte des réponses réelles.

\section{Questionnaire final}

Le questionnaire final de 20 minutes comprenait huit modules axés spécifiquement sur l'hypertension (tableau 1), des modules d'introduction et de conclusion (totalisant cinq minutes), ainsi qu'un module sur l'état de santé général. Le questionnaire complet est accessible sur le site Web de Statistique Canada (www. statcan.gc.ca/imdb-bmdi/instrument/5160_ Q4_V1-fra.htm). Les entrevues ont été réalisées à l'aide d'une application d'entrevue téléphonique assistée par ordinateur (ETAO), qui a facilité l'administration uniforme du questionnaire d'enquête. L'application d'ETAO a contrôlé l'enchaînement logique des questions, a précisé les plages de réponses valides, a fourni les valeurs minimales et maximales liées aux réponses quantitatives et les procédures normalisées en cas de nonréponse ${ }^{16}$. L'application a été soumise à des tests de bout en bout dans un environnement simulé de collecte de données.

\section{Population cible}

La population cible de l'EPMCC-H était constituée des adultes canadiens (20 ans et plus) ayant reçu un diagnostic d'hypertension, avec l'ESCC comme base de sondage. L'ESCC est une enquête transversale nationale qui a permis d'obtenir, auprès de la population canadienne, des données autodéclarées sur l'état de santé, l'utilisation des soins de santé et les déterminants de la santé depuis 2000 17-19. L'EPMCC-H a fourni des renseignements détaillés sur les personnes souffrant d'hypertension tout en permettant le jumelage avec l'ESCC principale, ce qui a permis d'obtenir des données supplémentaires sur les caractéristiques sociodémographiques et les facteurs de risque.

La population admissible à l'EPMCC-H de 2009 était constituée des Canadiens vivant dans un ménage privé de l'une des dix provinces. Les résidents des trois territoires du Nord n’ont pas été interrogés en raison de la petite taille de l'échantillon, qui aurait rendu impossible la pondération adéquate des résultats afin qu'ils représentent tous les résidents. Ont également été exclus de l'ESCC, et donc de l'EPMCC-H de 2009, les membres à temps plein des Forces canadiennes, la population vivant dans les réserves indiennes ou sur les terres de la Couronne, ainsi que les résidents d'établissements ou de certaines régions éloignées (représentant ensemble moins de $2 \%$ de la population cible) ${ }^{16,18}$.

Afin de cerner la population cible de l'EPMCC-H, on a utilisé un module standard de l'ESCC invitant les répondants à déclarer s'ils souffraient d'une maladie chronique, diagnostiquée par un professionnel de la santé et durant depuis six mois et plus. Les répondants âgés de 20 ans ou plus et ayant répondu « oui » à la question «Souffrez-vous d'hypertension artérielle? » ou « Au cours du dernier mois, avez-vous pris des médicaments pour l'hypertension? » (total de $\mathrm{n}=17$ 437) étaient admissibles ${ }^{16}$. Les femmes dont l'hypertension était associée à la grossesse ont été exclues. Seul le répondant à l'ESCC, et non tout le ménage, était considéré comme admissible. Les entrevues par procuration n'étaient pas permises.

\section{Méthode d'échantillonnage}

Des analyses de l'échantillon ont été effectuées à divers cycles de l'ESCC durant l'élaboration de l'EPMCC. Dans toutes ces analyses, la méthode d'échantillonnage par grappes à plusieurs degrés qui a été appliquée était similaire. Pour commencer, nous avons réparti les données brutes non pondérées de tous les répondants souffrant d'hypertension qui étaient disponibles dans divers domaines (sexe; groupe d'âge : 20-44 ans, 45-64 ans, 65-74 ans, 75 ans ou plus; province; région : Atlantique, Québec, Ontario, Prairies, Colombie-Britannique) et dans diverses combinaisons de domaines pour être sûrs de disposer de suffisamment de données finales dans ces domaines. L'administration de l'enquête à l'ensemble de cette population n'était pas réalisable. De plus, certains de ces répondants 
TABLEAU 1

Modules du questionnaire de l'EPMCC-H de 2009

\begin{tabular}{|c|c|c|c|c|}
\hline \multicolumn{2}{|c|}{ Module de l'EPMCC-H ${ }^{a}$} & \multirow{2}{*}{$\begin{array}{l}\text { Type de contenu } \\
\text { Administratif }\end{array}$} & \multirow{2}{*}{$\begin{array}{c}\begin{array}{c}\text { Nombre } \\
\text { de questions }\end{array} \\
0\end{array}$} & \multirow{2}{*}{$\begin{array}{l}\text { Brève description } \\
\text { Présente au répondant le contexte et l'objectif de l'enquête. }\end{array}$} \\
\hline 1 & Introduction de l'enquête & & & \\
\hline 2 & État de santé général & Général & 5 & $\begin{array}{l}\text { Invite le répondant à répondre à des questions au sujet de } \\
\text { son état de santé général avant de l'amener à des } \\
\text { questions axées spécifiquement sur l'hypertension. }\end{array}$ \\
\hline 3 & $\begin{array}{l}\text { Confirmation d'un diagnostic } \\
\text { d'hypertension }\end{array}$ & Axé sur l'hypertension & 5 & $\begin{array}{l}\text { Permet de confirmer que le répondant fait partie } \\
\text { de la population cible et invite le répondant à indiquer } \\
\text { l'âge qu'il avait au moment où il a reçu son diagnostic. }\end{array}$ \\
\hline 4 & Mesure de la pression artérielle & Axé sur l'hypertension & 9 & $\begin{array}{l}\text { Sert à obtenir de l'information sur la dernière fois } \\
\text { où la pression artérielle du répondant a été mesurée, } \\
\text { y compris la pression diastolique et la pression systolique } \\
\text { ainsi que les valeurs cibles, et à savoir si le répondant } \\
\text { dispose d'un plan pour contrôler sa pression artérielle. }\end{array}$ \\
\hline 5 & Consommation de médicaments & Axé sur l'hypertension & $9(10)^{c}$ & $\begin{array}{l}\text { Porte sur la pharmacothérapie dans son ensemble ainsi } \\
\text { que sur la pharmacothérapie contre l'hypertension, et } \\
\text { vise à déterminer l'observance du traitement. }\end{array}$ \\
\hline 7 & Recommandations cliniques & Axé sur l'hypertension & 8 & $\begin{array}{l}\text { Sert à documenter les recommandations précises formulées } \\
\text { au répondant par un professionnel de la santé pour l'aider } \\
\text { à contrôler sa pression artérielle. }\end{array}$ \\
\hline 8 & Autogestion & Axé sur l'hypertension & $14(22)^{d}$ & $\begin{array}{l}\text { Porte sur les recommandations que le répondant a tenté } \\
\text { de suivre, la mesure dans laquelle il prend en charge } \\
\text { sa condition et les obstacles auxquels il s'est heurté. }\end{array}$ \\
\hline 9 & Autosurveillance de la pression artérielle & Axé sur l'hypertension & 6 & $\begin{array}{l}\text { Porte sur la surveillance de la pression artérielle à } \\
\text { l'extérieur du cabinet du médecin et sur ce que signifient } \\
\text { pour le répondant les renseignements à ce sujet. }\end{array}$ \\
\hline 10 & Information et formation & Axé sur l'hypertension & 8 & $\begin{array}{l}\text { Concerne l'information au sujet de l'hypertension : source(s) } \\
\text { d'information, type de matériel/ressources accessibles, matériel/ } \\
\text { ressources dont le répondant préférerait disposer. }\end{array}$ \\
\hline
\end{tabular}

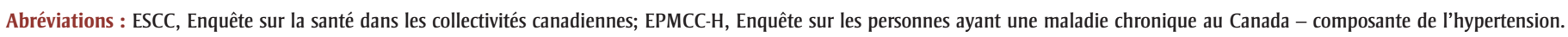

a Les 11 modules associés à l'EPMCC-H sont reliés à l'ESCC de 2008, ce qui représente en tout 87 modules permettant d'effectuer des analyses.

b Le nombre de questions posées à chaque répondant dépend de l'enchaînement des questions et de l'admissibilité du répondant aux différentes questions.

c Bien que ce module comprenne 9 questions, l'une d'elles comporte deux parties, ce qui donne en tout 10 questions.

${ }^{\mathrm{d}}$ Bien que ce module comprenne 14 questions, plusieurs d'entre elles comportent diverses parties, ce qui donne en tout 22 questions.

devaient participer à la composante de l'arthrite de l'EPMCC. Étant donné que les questionnaires des deux composantes de l'enquête devaient être administrés en même temps, les répondants choisis pour répondre au questionnaire d'une composante devenaient inadmissibles à l'autre composante, même s'ils souffraient des deux maladies. Ainsi, les données brutes ont été filtrées dans chaque domaine afin de créer un échantillon brut de répondants souffrant d'hypertension et admissibles à l'EPMCC-H. Durant ce processus, l'échantillon a été réparti en fonction des proportions relatives de cas d'arthrite et d'hypertension dans l'enquête principale, pour que la taille des cellules des deux enquêtes soit suffisante pour la réalisation d'analyses. Dans certains domaines, toutes les données brutes ont été conservées pour que l'échantillon soit suffisant.

Par la suite, l'échantillon brut a été ajusté à nouveau, cette fois en tenant compte de la perte d'échantillon probable. Le taux de réponse était estimé à $70 \%$, ce qui correspondait à environ $10 \%$ de perte en raison de l'échec du recrutement ou du refus d'autoriser le partage/jumelage des données, et $20 \%$ de perte en raison de l'absence de réponse. Un facteur d'ajustement de 0,70 a ainsi été appliqué à chaque domaine. Cela a permis d'obtenir le nombre de répondants souhaité pour l'administration du questionnaire d'enquête et a servi de base pour les analyses subséquentes de l'échantillon décrites ci-dessous.

$\mathrm{Au}$ démarrage de l'élaboration de l'EPMCC, nous avons utilisé le fichier de 
l'ESCC de 2005 pour évaluer la possibilité d'obtenir des échantillons suffisants pour mener simultanément les enquêtes sur l'arthrite et l'hypertension. Les analyses ont été centrées sur l'estimation de la taille minimale de l'échantillon nécessaire pour produire des estimations fiables pour chaque domaine. Pour l'hypertension, il fallait disposer d'un échantillon d'au moins 1324 répondants, en utilisant comme hypothèse un effet du plan de sondage fixé à 2,8 pour le groupe d'âge et le sexe, étant donné que la variance de l'échantillon était environ 2,8 fois plus élevée qu'elle ne l'aurait été si l'enquête avait été fondée sur une sélection aléatoire. Pour la province et la région, l'effet du plan de sondage a été fixé à 3 . Les résultats de cette analyse de l'échantillon ont confirmé que les populations disponibles étaient suffisantes pour mener des enquêtes indépendantes sur l'arthrite et l'hypertension.

Peu avant d'administrer l'enquête, nous avons utilisé le fichier de l'ESCC de 2007 pour évaluer la possibilité de publication des résultats. Le but était de déterminer le taux de prévalence minimal requis, par domaine, pour obtenir un coefficient de variation (CV) préétabli de 16,5\%. Bien que le $\mathrm{CV}$ maximal soit généralement de $33,3 \%$, seuil au-delà duquel les données seraient considérées comme non publiables, le CV ciblé, plus conservateur (égal ou inférieur à 16,5\%), garantissait des estimations fiables. Un tel CV rendait les estimations fiables pour la plupart des groupes d'âge et selon le sexe, mais seules les estimations nationales ou régionales étaient publiables.

Enfin, pour déterminer la population admissible à l'EPMCC-H de 2009, nous avons choisi les répondants à partir des données vérifiées du fichier de l'ESCC de 2008. Le nombre de répondants admissibles sélectionnés a été augmenté autant que possible (d'environ 6000 à 9055 répondants) afin d'atténuer l'effet des non-réponses et des cas excédant la portée de l'enquête. D'autres renseignements, dont la répartition de l'échantillon admissible par domaine, sont disponibles à l'adresse http://www.statcan.gc.ca/imdbbmdi/document/5160_D5_T1_V1-fra.htm.

\section{Recrutement, collecte des données et traitement}

Le recrutement en vue de l'EPMCC-H de 2009 a commencé à la mi-janvier 2009, avec l'envoi par la poste de lettres de présentation aux répondants sélectionnés, suivi par des entrevues téléphoniques. Différentes mesures ont été prises afin de maximiser le taux de réponse, notamment l'envoi de ces lettres par la poste, le fait d'offrir au répondant la possibilité de choisir un moment qui lui convenait pour l'entrevue, le dépistage des répondants ayant déménagé ou dont les numéros de téléphone étaient invalides, et le fait de mener l'entrevue en français ou en anglais, selon le choix du répondant ${ }^{16}$. Les intervieweurs devaient communiquer aux répondants le titre de l'enquête, son but et la loi en vertu de laquelle elle était menée, le caractère volontaire de la participation et le fait que les renseignements personnels seraient protégés. Les répondants devaient donner leur consentement verbal éclairé pour participer.

La collecte des données a commencé le 3 février 2009 et a duré trois mois. Entre avril et décembre 2009, les données ont été soumises à des processus de traitement, d'estimation et de documentation. Dans le cas des répondants ayant autorisé le jumelage et le partage de leurs données, les renseignements fournis dans le cadre de l'EPMCC-H de 2009 ont été couplés à ceux de l'ESCC de 2008. Afin d'en protéger la confidentialité, toutes les données d'identification personnelle ont été supprimées du fichier de partage-jumelage. Les données étaient prêtes en décembre 2009 et ont été transmises à l'ASPC, à Santé Canada et aux ministères provinciaux de la Santé. Les chercheurs et les tiers peuvent accéder aux fichiers principaux par l'intermédiaire des Centres de données de recherche dirigés par Statistique Canada (http://www.statcan .gc.ca/rdc-cdr/process-fra.htm).

\section{Analyse des données}

Afin que les estimations soient représentatives de la population cible, des poids d'enquête ont été produits. Fondées sur l'échantillon final de l'EPMCC-H, les valeurs des poids correspondaient au nombre de personnes faisant partie de la population canadienne représentée par chaque répondant. Les poids d'enquête et les répliques bootstrap ont été rajustés afin de prendre en compte les cas excédant la portée de l'enquête, les non-réponses et les cas où le répondant refusait que ses données soient partagées ${ }^{16,20}$. Afin de comparer, entre les deux enquêtes, les caractéristiques des répondants souffrant d'hypertension, la population de l'ESCC de 2008 a été limitée aux adultes de 20 ans et plus et les résidents des territoires et les femmes enceintes ont été exclus. Les estimations ont été pondérées à l'aide des poids appropriés pour chaque enquête, et la méthode de rééchantillonnage bootstrap a été utilisée pour déterminer les intervalles de confiance (IC) à l'aide de SAS Enterprise Guide, version 4 (SAS Institute Inc., Cary, Caroline du Nord, É.-U.). La diffusion des données a été soumise aux lignes directrices en matière de qualité des données énoncées par Statistique Canada pour ce qui est de l'arrondissement et des erreurs dues à l'échantillonnage ${ }^{16}$.

\section{Résultats}

\section{Population de l'échantillon final}

La figure 1 illustre la participation des répondants à l'EPMCC-H de 2009. Un total de 17437 répondants ayant déclaré avoir reçu un diagnostic d'hypertension dans le cadre de l'ESCC de 2008 a constitué la base de sondage. La perte prévue entre la base de sondage et l'échantillon final de l'EPMCC-H de 2009 reposait sur des critères d'exclusion préétablis (répondants de moins de 20 ans, résidents des territoires, femmes souffrant uniquement d'hypertension associée à la grossesse) et sur des personnes contactées se révélant être des cas hors du champ de l'enquête (décès, déménagement, faux positif, faux négatif). En réalité, la proportion de cas hors du champ de l'EPMCC-H (13 \%) a dépassé les estimations prévues (10\%), en grande partie en raison de la classification inappropriée des répondants. Un résultat était considéré comme un faux positif si le répondant initialement inclus dans la population cible de l'EPMCC-H déclarait par la suite ne pas souffrir d'hypertension, ce qui pouvait notamment 
FIGURE 1

Participation des répondants à l'EPMCC-H de $2009^{\mathrm{a}}$

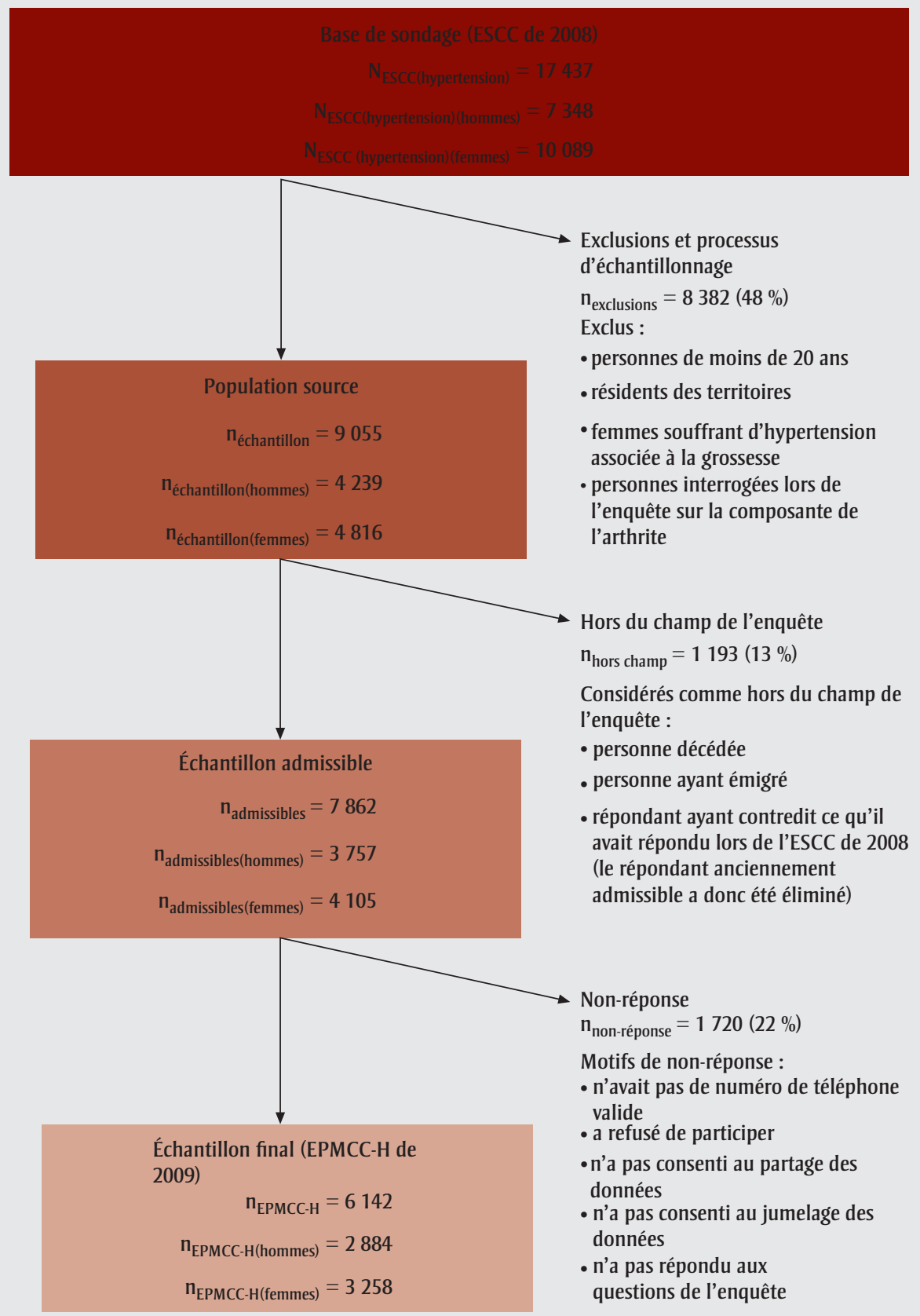

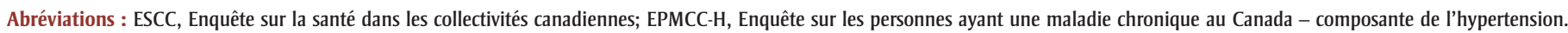

${ }^{a}$ Chiffres non pondérés.

relever de l'insistance à ce que le diagnostic ait été posé par un professionnel de la santé, éliminant ainsi les personnes ayant diagnostiqué elles-mêmes leur maladie ou ayant mal interprété la question initiale. Les faux négatifs ont pu provenir d'une perte de répondants souffrant réellement d'hypertension, mais ayant répondu «non » aux questions de sélection, certains participants pouvant avoir répondu ainsi pour diverses raisons, notamment pour éviter de participer à l'EPMCC-H.

$\mathrm{Au}$ cours du processus d'élaboration, d'administration et de traitement de l'enquête, certains répondants admissibles ont également pu être écartés s'ils avaient été inclus dans l'échantillon de la composante de l'arthrite, s'ils ne voulaient pas que l'on communique avec eux après qu'ils eurent répondu à l'ESCC de 2008, s'ils étaient absents à plusieurs reprises au moment de l'entrevue, s'ils refusaient de 
répondre à l'enquête ou s'ils refusaient que leurs données soient couplées ou utilisées. Le taux de succès, c'est-à-dire le nombre de personnes faisant partie de l'échantillon admissible et ayant été interrogées ( $n=7862$ ) en tant que proportion de la population source ( $\mathrm{n}=9$ 055), variait chez les hommes de $75,2 \%$ pour les 20-44 ans à 93,1 \% pour les $65-74$ ans $^{16}$. De manière similaire, chez les femmes, le taux de succès le plus faible était chez les répondantes des groupes d'âge inférieurs $(51,1 \%)$ et le plus élevé dans le groupe des 65-74 ans $(94,7 \%)^{16}$. Le taux de réponse, c'est-à-dire l'échantillon final de répondants à l'enquête $(n=6142)$ par rapport à l'échantillon admissible ( $\mathrm{n}=7$ 862), variait également, passant d'un taux faible dans le groupe des 20-44 ans (hommes : 65,6\%; femmes : $71,7 \%$ ) à un taux élevé dans le groupe des 65-74 ans (hommes : 79,7\%; femmes : $82,1 \%)^{16}$. L'échantillon final disponible pour l'analyse comprenait 6142 répondants, soit un taux de réponse global de 78,1 \%.

\section{Caractéristiques de la population}

Le tableau 2 présente une comparaison de certaines caractéristiques sociodémographiques et liées à la santé des répondants de 20 ans et plus qui avaient déclaré souffrir d'hypertension dans le fichier partagé de l'ESCC de 2008 et des répondants à l'EPMCC-H de 2009. L'échantillon de l'EPMCC-H de 2009 était représentatif de la population de l'ESCC pour ce qui est de l'origine ethnique, de l'indice de masse corporelle, du tabagisme, du diabète autodéclaré, de l'accès à un médecin régulier et du nombre de consultations médicales au cours de la dernière année. Quelques indicateurs étaient significativement différents (c'est-à-dire valeur de $p<0,05$ ou IC ne se chevauchant pas). Pour ce qui est de l'EPMCC-H, l'âge moyen de la population était de 61,2 ans (IC à $95 \%$ : 60,8 à 61,6) alors qu'il était de 62,2 ans (IC à $95 \%$ : 61,8 à 62,5) pour l'ESCC de 2008, une proportion plus élevée de répondants détenaient un diplôme d'études postsecondaires (EPMCC-H : 52,0 \%, IC à $95 \%: 49,7 \%$ à $54,2 \%$; ESCC : 47,5 \%, IC à $95 \%: 46,1 \%$ à $48,9 \%$ ) et une proportion plus faible de répondants ont déclaré prendre des médicaments pour contrôler l'hypertension (EPMCC-H : 82,5\%, IC à $95 \%$ : 80,9 \% à
84,1 \% ; ESCC : 88,6 \%, IC à $95 \%$ : 87,7 \% à $89,6 \%)$. Des différences significatives fondées sur une valeur de $p$ inférieure à 0,05 ont été observées pour certaines catégories relevant d'autres variables, notamment le sexe masculin, l'état de santé autoévalué considéré comme mauvais/ passable, le niveau d'activité physique considéré comme actif, le revenu ainsi que les maladies du coeur et les AVC autodéclarés. Cependant, dans ces cas, les IC se chevauchaient et le rapport des proportions était proche de 1 (variant de $0,87$ à 1,27$)$.

\section{Caractéristiques de la participation au sondage}

Une analyse de la fréquence non pondérée a révélé que la plupart des questions avaient moins de $1 \%$ de données manquantes (non présentées). Même si les options " ne sait pas » (NSP) et "refus » (R) étaient offertes pour la plupart des questions, ces catégories de réponses n'étaient pas lues à voix haute. Les questions dont la prévalence des réponses NSP, R ou " non précisé » était plus élevée étaient associées à certains sujets. Par exemple, on demandait aux répondants d'indiquer leur pression systolique et leur pression diastolique. On s'attendait à ce que les répondants aient de la difficulté à fournir ces données, et respectivement $18,0 \%$ et $22,3 \%$ des répondants n'ont pas fourni de réponse valide quant à leur pression systolique et diastolique. Néanmoins, ces questions étaient intentionnellement posées afin d'offrir une information de base sur la sensibilisation et les connaissances de la population à propos de l'hypertension.

Dans la plupart des cas, les échelles de réponse et la répartition par catégorie étaient raisonnables. Toutefois, pour certaines questions concernant l'état de santé général dont l'échelle de réponse comprenait cinq catégories (" excellent», " très bon ", "bon ", " passable » et " mauvais »), la réponse la plus fréquente était " bon ", ce qui représente une tendance centrale. Cela laisse croire que le format de certaines échelles pourrait avoir contribué à l'obtention de réponses neutres.

\section{Analyse}

L'enquête par sondage présentée ici englobe un grand nombre de questions touchant les Canadiens souffrant d'hypertension, comme la connaissance des niveaux de pression artérielle, les pratiques en matière d'autosurveillance, les recommandations cliniques, la pharmacothérapie ainsi que les stratégies d'autogestion et les obstacles à l'autogestion. L'EPMCC-H a déjà permis la production de plusieurs résultats et l'établissement d'un profil fiable des Canadiens souffrant d'hypertension.

Plus précisément, l'enquête a permis de conclure que le taux d'utilisation des médicaments pour l'hypertension était élevé au Canada $(82,5 \%$ des adultes souffrant d'hypertension), et que $10 \%$ des personnes hypertendues contrôlaient leur hypertension en modifiant seulement leur mode de $v^{21,22}$. Parmi ceux qui prenaient des médicaments pour l'hypertension, ni l'augmentation du nombre de médicaments ni la fréquence de la prise des médicaments n'ont été associées à une non-observance du traitement ${ }^{21}$. Un nombre impressionnant de répondants - la majorité - ont déclaré avoir recours à des stratégies fondées sur la modification du mode de vie, mais moins de la moitié ont indiqué appliquer ces mesures de façon constante, et une proportion déconcertante de répondants ont indiqué que leur professionnel de la santé ne leur fournissait pas de conseils à propos des diverses stratégies axées sur la modification du mode de vie ${ }^{22-24}$. En outre, Gee et collab. ${ }^{24}$ ont observé que les obstacles à l'abandon des comportements négatifs pour la santé différaient des obstacles à l'adoption de comportements sains.

Nous avons généré les profils des sousgroupes à risque plus élevé, dont les personnes qui risquent de ne pas adopter de stratégie visant à modifier leur mode de vie ou celles pour qui la probabilité de surveiller leur pression artérielle à l'extérieur du cabinet du médecin est moins élevée ${ }^{24,25}$. Divers impacts négatifs ont été associés au fait qu'un répondant croie avoir peu de contrôle sur son hypertension et au fait qu'un professionnel de la santé ne fournisse pas de conseils ou de 
TABLEAU 2

Comparaison des caractéristiques de la population source souffrant d'hypertension (ESCC de 2008) et de celles des répondants à l'EPMCC-H de 2009

\begin{tabular}{|c|c|c|c|c|c|c|}
\hline & \multicolumn{4}{|c|}{ Population souffrant d'hypertension, 20 ans et plus } & \multirow[t]{3}{*}{ valeur de $p^{d}$} & \multirow{3}{*}{$\begin{array}{c}\text { Ratio } \\
\text { ESCC:EPMCC-H }\end{array}$} \\
\hline & \multicolumn{2}{|c|}{$\begin{array}{l}\text { ESCC de } 2008 \\
\left(n=13896^{a}\right)\end{array}$} & \multicolumn{2}{|c|}{$\begin{array}{l}\text { EPMCC-H de } 2009 \\
(n=6 \text { 142) }\end{array}$} & & \\
\hline & $n^{\mathbf{b}}$ & \%c (IC à $95 \%$ ) & $n^{\mathbf{b}}$ & \%c (IC à $95 \%$ ) & & \\
\hline \multicolumn{7}{|l|}{ Sexe } \\
\hline Homme & 5961 & $48,2(47,1$ à 49,4$)$ & 2884 & $46,7(45,1$ à 48,4$)$ & $0,03^{\mathrm{e}}$ & 1,03 \\
\hline \multicolumn{7}{|l|}{ Âge } \\
\hline 45 à 64 ans & 5411 & 45,4 (44,1 à 46,7) & 2025 & 48,0 (46,2 à 49,8) & $0,0009^{\mathrm{e}}$ & 0,95 \\
\hline 65 ans et plus & 7503 & $44,5(43,4$ à 45,6$)$ & 3484 & $40,8(39,2$ à 42,4) & $<0,0001^{\mathrm{f}}$ & 1,09 \\
\hline Moyenne & & $62,2(61,8$ à 62,5) & & 61,2 (60,8 à 61,6) & $<0,0001^{f}$ & 1,02 \\
\hline \multicolumn{7}{|l|}{ Origine ethnique } \\
\hline Blanc & 12535 & $85,3(83,8$ à 86,8$)$ & 5676 & $86,8(84,6$ à 89,0$)$ & 0,13 & 0,98 \\
\hline Inférieur au diplôme d'études secondaires & 4419 & $25,9(24,7$ à 27,2$)$ & 1798 & $23,3$ (21,5 à 25,1$)$ & $0,001^{\mathrm{e}}$ & 1,11 \\
\hline Diplôme d'études secondaires & 2170 & $16,8(15,7$ à 17,9$)$ & 961 & 17,6 (15,7 à 19,4) & 0,37 & 0,95 \\
\hline Études postsecondaires & 772 & $6,2(5,4$ à 6,8) & 358 & $7,2(5,9$ à 8,4$)$ & 0,06 & 0,86 \\
\hline Diplôme d'études postsecondaires & 6177 & $47,5(46,1$ à 48,9$)$ & 2988 & $52,0(49,7$ à 54,2$)$ & $<0,0001^{\mathrm{f}}$ & 0,91 \\
\hline \multicolumn{7}{|l|}{ Revenu total du ménage } \\
\hline Moins de $15000 \$$ & 1247 & $7,1(6,4$ à 7,8$)$ & 473 & $6,1(5,0$ à 7,2$)$ & $0,04^{e}$ & 1,16 \\
\hline 15000 à $29999 \$$ & 3106 & $19,4(18,3$ à 20,5$)$ & 1410 & $19,5(17,7$ à 21,4$)$ & 0,85 & 0,99 \\
\hline 30000 à 49999 \$ & 2846 & $21,9(20,7$ à 23,1$)$ & 1351 & 20,0 (18,2 à 21,7) & $0,02^{\mathrm{e}}$ & 1,10 \\
\hline 50000 à $79999 \$$ & 2526 & $24,8(23,2$ à 26,3$)$ & 1255 & 23,7 (21,6 à 25,8) & 0,29 & 1,05 \\
\hline \multicolumn{7}{|l|}{ IMC $\left(\mathbf{k g} / \mathrm{m}^{2}\right)$} \\
\hline Moins de 25 (sous la normale/poids santé) & 3873 & $29,8(28,4$ à 31,2$)$ & 1792 & 28,5 (26,5 à 30,6) & 0,17 & 1,05 \\
\hline 25 à 29 (embonpoint) & 5103 & 39,3 (37,8 à 40,8) & 2415 & 38,4 (36,1 à 40,8) & 0,42 & 1,02 \\
\hline 30 et plus (obésité) & 4098 & $30,9(29,6$ à 32,3$)$ & 1805 & 33,0 (30,6 à 35,4) & 0,05 & 0,94 \\
\hline \multicolumn{7}{|l|}{ Niveau d'activité physique } \\
\hline Actif & 2286 & $16,8(15,8$ à 17,8$)$ & 1177 & $18,5$ (16,9 à 20,1$)$ & $0,02^{e}$ & 0,91 \\
\hline Modérément actif & 3157 & $22,8(21,6$ à 23,9) & 1490 & $23,2(21,3$ à 25,0$)$ & 0,61 & 0,98 \\
\hline Inactif & 8022 & $56,8(55,4$ à 58,2$)$ & 3472 & 58,4 (56,2 à 60,5) & 0,11 & 0,97 \\
\hline \multicolumn{7}{|l|}{ Tabagisme } \\
\hline Fumeur quotidien & 1984 & $14,1(13,2$ à 15,0$)$ & 842 & $14,0(12,5$ à 15,5$)$ & 0,90 & 1,01 \\
\hline Fumeur occasionnel & 311 & 2,3 (1,8 à 2,8) & 149 & $3,1(2,1$ à 4,2$)$ & 0,08 & 0,74 \\
\hline Non-fumeur & 11564 & $83,2(82,2$ à 84,3$)$ & 5149 & 82,9 (81,1 à 84,7) & 0,64 & 1,00 \\
\hline \multicolumn{7}{|l|}{ Comorbidités } \\
\hline Diabète & 2830 & $20,3(19,1$ à 21,5$)$ & 1172 & $19,2(17,0$ à 21,3$)$ & 0,21 & 1,06 \\
\hline Maladies du coeur & 2590 & $16,3(15,4$ à 17,3$)$ & 1077 & 14,7 (13,0 à 16,4) & $0,03^{\mathrm{e}}$ & 1,11 \\
\hline Séquelles d'un AVC & 627 & 3,8 (3,3 à 4,2) & 223 & $3,0(2,4$ à 3,6$)$ & $0,006^{\mathrm{e}}$ & 1,27 \\
\hline
\end{tabular}


TABLEAU 2 (Suite)

Comparaison des caractéristiques de la population source souffrant d'hypertension (ESCC de 2008) et de celles des répondants à l'EPMCC-H de 2009

\begin{tabular}{|c|c|c|c|c|c|c|}
\hline & \multicolumn{4}{|c|}{ Population souffrant d'hypertension, 20 ans et plus } & \multirow[t]{3}{*}{ valeur de $p^{d}$} & \multirow{3}{*}{$\begin{array}{c}\text { Ratio } \\
\text { ESCC:EPMCC-H }\end{array}$} \\
\hline & \multicolumn{2}{|c|}{$\begin{array}{l}\text { ESCC de } 2008 \\
\left(n=13896^{a}\right)\end{array}$} & \multicolumn{2}{|c|}{$\begin{array}{c}\text { EPMCC-H de } 2009 \\
(n=6 \text { 142) }\end{array}$} & & \\
\hline & $n^{b}$ & $\%^{\mathrm{c}}$ (IC à $\left.95 \%\right)$ & $n^{b}$ & $\%^{\mathrm{c}}$ (IC à $\left.95 \%\right)$ & & \\
\hline \multicolumn{7}{|l|}{ Soins médicaux } \\
\hline A un médecin de famille & 13179 & $94,9(94,3$ à 95,6$)$ & 5825 & $95,1(94,2$ à 96,0$)$ & 0,72 & 1,00 \\
\hline Prend des médicaments pour l'hypertension & 12717 & $88,6(87,7$ à 89,6$)$ & 5171 & $82,5(80,9$ à 84,1$)$ & $<0,0001^{\mathrm{f}}$ & 1,07 \\
\hline
\end{tabular}

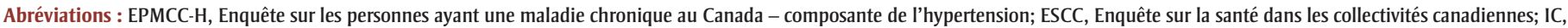
intervalle de confiance; IMC, indice de masse corporelle.

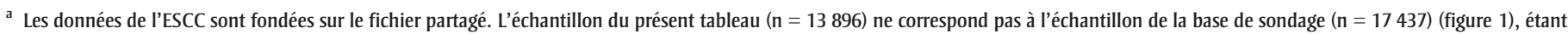
donné que des critères d'exclusion ont été appliqués ici (moins de 20 ans, résidents des territoires, femmes souffrant d'hypertension associée à la grossesse); par ailleurs, ici on inclut les personnes souffrant d'arthrite, alors que dans la figure 1, certaines personnes interrogées peuvent avoir été retirées ultérieurement en vue de la composante de l'arthrite.

b Chiffres non pondérés.

c Les proportions sont fondées sur des chiffres pondérés afin de refléter la population canadienne vivant dans les dix provinces.

${ }^{\text {d }}$ Les valeurs de $p$ ont été établies à l'aide de tests $z$ afin de déterminer les différences statistiquement significatives entre les deux ratios.

e Différences statistiquement significatives si $p<0,05$. Cependant, il convient de noter que les IC se chevauchent et que la différence entre les populations est faible.

${ }^{\mathrm{f}}$ Différences statistiquement significatives si $p<0,05$; les IC ne se chevauchent pas.

renseignements sur la gestion du mode de $v^{23,26}$. Ce type de résultat pourrait orienter des interventions ciblées.

Dans l'ensemble, l'EPMCC-H de 2009 est représentative de sa population source, bien que les répondants à l'EPMCC-H soient plus jeunes et davantage scolarisés, et qu'il soit moins probable qu'ils suivent un traitement antihypertenseur. Ce biais potentiel de sélection pourrait notamment faire en sorte que les données représentent un groupe chez qui le diagnostic est plus récent, peut-être plus en santé, souffrant d'hypertension pendant des périodes plus courtes. En se fondant uniquement sur les valeurs de $p$, d'autres différences sont aussi statistiquement significatives, bien que les IC se chevauchaient et que l'ampleur relative d'une proportion comparée à une autre équivalait presque à 1 . En résumé, malgré le fait que les valeurs de $p$ soient statistiquement significatives, il n'existe peut-être pas de différences importantes, et il revient aux utilisateurs de déterminer si cela a une incidence sur leurs analyses.

\section{Forces et limites}

D’un point de vue plus général, l'EPMCC-H de 2009 a été élaborée afin d'être repré- sentative à l'échelle nationale. Cependant, la représentativité des données pour la population canadienne pourrait être limitée en raison de l'exclusion des territoires et de certaines populations. Selon les données administratives, le taux d'incidence normalisé selon l'âge de l'hypertension au Yukon est très supérieur à la moyenne canadienne (37,7 par 1000 habitants contre 25,8), mais le taux de prévalence normalisé selon l'âge est inférieur $(17,9 \% \text { contre } 19,6 \%)^{1,2}$. Il serait intéressant d'étudier le diagnostic et la prise en charge de l'hypertension au Yukon. De plus, d'autres populations qui ont pu être exclues (p. ex. certains groupes ethniques) auraient probablement présenté des caractéristiques différentes $^{27}$. Le fait que l'EPMCC-H ait été administrée en deux langues seulement a peut-être causé l'exclusion de certains des 493 (1,7 \%; données non pondérées) participants qui avaient initialement répondu à l'ESCC de 2008 dans une langue autre que l'anglais ou le français. Pour les enquêtes à venir, le suréchantillonnage des populations vulnérables ou ethniques est donc encouragé.

Il est bien connu que les enquêtes fondées sur des données fournies par les répondants sont limitées parce qu'elles peuvent être sujettes à diverses erreurs, liées ou non à l'échantillonnage, par exemple un biais lié aux réponses, un biais de rappel ou une erreur de classification non différentielle. Étant donné que l'enquête visait à comprendre la façon dont les personnes souffrant d'hypertension prennent en charge leur maladie, la population cible était composée de personnes ayant déclaré avoir reçu un diagnostic d'hypertension et excluait les personnes atteintes d'hypertension non diagnostiquée. Bien que la majorité des Canadiens souffrant d'hypertension (83\%) soient au courant de leur condition $^{3}$, l'exactitude de l'état autodéclaré quant à l'hypertension demeure incertaine. Nous avons vu que des personnes n'ayant reçu aucun diagnostic peuvent avoir déclaré souffrir de la maladie (faux positif), tandis que des personnes dont l'hypertension est contrôlée peuvent déclarer ne pas souffrir de la maladie (faux négatif). Cependant, le taux d'erreur de classification est probablement inférieur dans l'EPMCC-H, compte tenu du fait que ces cas ont été repérés au cours du processus de sélection.

On a tenté de déterminer si les changements apportés au mode de vie étaient attribuables au fait d'avoir reçu un diagnostic d'hypertension. En fait, la mo- 
dification du mode de vie peut être influencée par de nombreux facteurs autres qu'un diagnostic. Cette enquête présente en outre une autre limite : en effet, bien que le jumelage avec l'ESCC ait permis d'obtenir d'autres variables et d'améliorer l'efficacité de l'enquête, les caractéristiques des participants peuvent avoir changé entre le moment où les deux enquêtes ont été menées (moyenne de $8,5$ mois $)^{26}$, ce qui peut avoir entraîné des erreurs de classification. Statistique Canada a pris certaines mesures afin de réduire les erreurs relatives aux enquêtes, par exemple en utilisant le système d'ETAO et en offrant une formation approfondie aux intervieweurs afin de réduire au minimum le taux de nonréponse. Dans le cas de l'EPMCC-H en particulier, l'Institut de recherche en santé Lawson a lancé une étude de validation afin de comparer, au moyen de la méthode de test-retest, les réponses fournies par deux populations souffrant d'hypertension.

\section{Conclusion}

L'EPMCC-H de 2009 fournit des données nouvelles et exhaustives sur le diagnostic de l'hypertension et les mécanismes de prise en charge utilisés par les Canadiens ayant déclaré présenter une pression artérielle élevée. Compte tenu du succès remporté par la première version de l'EPMCC, la méthodologie et le contenu ont depuis été adaptés pour deux autres cycles de l'enquête (diabète et asthme/ maladie pulmonaire obstructive chronique), dont les données ont été publiées en $2011^{28}$. La méthodologie a également été adaptée pour l'Enquête sur les personnes ayant des problèmes neurologiques au Canada, dont les données ont été publiées à la fin de $2012^{29}$. Ces données pourraient créer des occasions de mener de nouvelles recherches, d'influencer l'élaboration des politiques et d'orienter les stratégies visant à améliorer la prévention et le contrôle des maladies chroniques au Canada.

\section{Remerciements}

Les auteurs déclarent n'avoir aucun conflit d'intérêts. Aucun financement de l'extérieur n'a été obtenu pour cette étude.
L'enquête a été parrainée et élaborée par l'Agence de la santé publique du Canada (D ${ }^{\text {re }}$ Paula Stewart, $\mathrm{M}^{\mathrm{me}}$ Asako S. Bienek, M. Jay Onysko, $\mathrm{D}^{\text {re }}$ Christina Bancej, $M^{\text {me }}$ Deirdre MacGuigan et $M^{\text {me }}$ Marianne E. Gee) en collaboration avec Statistique Canada (M. Mamadou S. Diallo, $\mathrm{M}^{\text {me }}$ Christy da Silva, $\mathrm{M}^{\mathrm{me}}$ Stacey Wan, $\mathrm{M}$. Sylvain Tremblay, M. Vince Dale, $\mathrm{M}^{\mathrm{me}}$ Cathy Trainor, $\mathrm{M}^{\mathrm{me}}$ Cindy Bennett, $\mathrm{M}^{\text {me }}$ Marie-Noëlle Parent et $\mathrm{M}^{\text {me }}$ Brenda Bélanger).

L’EPMCC-H a été élaborée grâce à la contribution en temps et à l'expertise des membres du groupe de travail externe, soit $D^{\mathrm{r}}$ Norm R. C. Campbell (Programme éducatif canadien sur l'hypertension), $\mathrm{D}^{\mathrm{re}}$ Femida Gwadry-Sridhar (Université Western Ontario), $\mathrm{M}^{\mathrm{me}}$ Robin L. Walker (Université de Calgary), $\mathrm{D}^{\mathrm{r}}$ Janusz Kaczorowski (Université de Montréal), $\mathrm{D}^{\mathrm{r}}$ Michel Joffres (Université Simon Fraser), $D^{r}$ Robert P. Nolan (University Health Network et Université de Toronto), $\mathrm{D}^{\mathrm{r}}$ Patrice Lindsay (Réseau canadien contre les accidents cérébrovasculaires) et $\mathrm{D}^{\mathrm{r}}$ Hude Quan (Université de Calgary).

\section{Références}

1. Agence de la santé publique du Canada. Rapport du Système national de surveillance des maladies chroniques : l'hypertension au Canada, 2010, 2010 [Internet]. Ottawa (Ont.) : Division de la surveillance, Centre de prévention et de contrôle des maladies chroniques; 2011 [consulté le 3 mars 2011]. PDF (1,81 Mo) téléchargeable à partir du lien : http://www.phac-aspc.gc.ca /cd-mc/cvd-mcv/ccdss-snsmc-2010/pdf/ CCDSS_HTN_Report_FINAL_FR_20100513 .pdf

2. Robitaille C, Dai S, Waters C et collab. Diagnosed hypertension in Canada: incidence, prevalence, and associated mortality. CMAJ. 2012 Jan;184(1):E49-56.

3. Wilkins K, Campbell NR, Joffres MR et collab. Tension artérielle des adultes au Canada. Rapp. sur la santé. 2010 Mar;21(1):37-46
4. Rabi DM, Daskalopoulou SS, Padwal RS et collab. The 2011 Canadian Hypertension Education Program recommendations for the management of hypertension : blood pressure measurement, diagnosis, assessment of risk, and therapy. Can J Cardiol. 2011 Jul-Aug;27 (4):415-33.e1-2.

5. Statistique Canada. Autres périodes de référence - Enquête sur la santé dans les collectivités canadiennes - Composante annuelle [Internet]. Ottawa (Ont.) : Statistique Canada; 2011 [consulté le 25 mars 2011]. Consultable à la page : http:// www23.statcan.gc.ca/imdb/p2SV_f.pl ? Function $=$ getInstanceList $\&$ SurvId $=3226$ $\&$ SurvVer $=1 \&$ InstaId $=15282 \&$ SDDS $=3226$ \&lang $=\mathrm{en} \& \mathrm{db}=\mathrm{imdb} \& \mathrm{adm}=8 \&$ dis $=2$

6. Statistique Canada. Enquête nationale sur la santé de la population - Volet ménages Longitudinal (ENSP) [Internet]. Ottawa (Ont.) : Statistique Canada; 2011 [mis à jour le 20 juillet 2011; consulté le 23 mars 2011]. Consultable à la page : http://www23 .statcan.gc.ca/imdb/p2SV_f.pl?Function $=$ getSurvey $\&$ SDDS $=3225 \&$ lang $=$ en $\& d b=$ imdb\&adm $=8 \&$ dis $=2$

7. National Health and Nutrition Examination Survey - NHANES 2005-2006 [Internet]. Atlanta (GA) : Centers for Disease Control and Prevention; 2011 [mis à jour le 29 avril 2011; consulté le 23 mars 2011]. Consultable à la page : http://www .cdc.gov/nchs/nhanes/nhanes2005-2006/ nhanes05_06.htm

8. Moser M, Franklin SS. Hypertension management : results of a new national survey for the hypertension education foundation: Harris interactive. J Clin Hypertens. 2007 May;9(5):316-23.

9. Reis JP, Dubose KD, Ainsworth BE, Macera CA, Yore MM. Reliability and validity of the occupational physical activity questionnaire. Med Sci Sports Exerc. 2005 Dec;37(12):2075-83.

10. Morisky DE, Green LW, Levine DM. Concurrent and predictive validity of a self-reported measure of medication adherence. Med Care. 1986 Jan;24(1):67-74. 
11. Grace SL, Barry-Bianchi S, Stewart DE, Rukholm E, Nolan RP. Physical activity behavior, motivational readiness and selfefficacy among Ontarians with cardiovascular disease and diabetes. J Behav Med. 2007 Feb;30(1):21-9. Epub 2006 Nov 16.

12. Quinn RR, Hemmelgarn BR, Padwal RS et collab. The 2010 Canadian Hypertension Education Program recommendations for the management of hypertension: part I blood pressure measurement, diagnosis, and assessment of risk. Can J Cardiol. 2010 May;26(5):241-8.

13. Hackam DG, Khan NA, Hemmelgarn BR et collab. The 2010 Canadian Hypertension Education Program recommendations for the management of hypertension : part 2 therapy. Can J Cardiol. 2010 May;26(5): 249-58.

14. National Heart, Lung, and Blood Institute. The seventh report of the Joint National Committee on prevention, detection, evaluation, and treatment of high blood pressure (JNC7) [Internet]. Bethesda (MD) : U.S. Department of Health and Human Services; 2003 [consulté le 23 mars 2011]. Consultable à la page : http://www .nhlbi.nih.gov/guidelines/hypertension/

15. National Heart, Lung, and Blood Institute. Third report of the Expert Panel on detection, evaluation, and treatment of high blood cholesterol in adults (adult treatment panel III) [Internet]. Bethesda (MD) : U.S. Department of Health and Human Services; 2003 [consulté le 23 mars 2011]. Consultable à la page : http://www.nhlbi .nih.gov/guidelines/cholesterol/

16. Statistique Canada. Documentation Enquête sur les personnes ayant une maladie chronique au Canada - Guide de l'utilisateur - 2009 [Internet]. 2010 [mis à jour le 29 novembre 2011; consulté le 12 avril 2011]. Consultable à la page : http://www23.statcan .gc.ca/imdb-bmdi/pub/document/5160_D5 _T1_V1-fra.htm

17. Statistique Canada. Enquête sur la santé dans les collectivités canadiennes Composante annuelle (ESCC) [Internet]. Ottawa (Ont.) : Statistique Canada; 2010 [mis à jour le 9 juin 2011; consulté le 3 mars 2011]. Consultable à la page : http: //www23.statcan.gc.ca/imdb/p2SV_f.pl ?Function $=$ getSurvey $\&$ SDDS $=3226 \&$ lang $=\mathrm{en} \& \mathrm{db}=\mathrm{imdb} \& \mathrm{adm}=8 \& \mathrm{dis}=2$
18. Béland Y. Enquête sur la santé dans les collectivités canadiennes - Aperçu de la méthodologie. Rapp. sur la santé. 2002 Mar;13(3):9-14.

19. Desmeules M. Appendix A : overview of National Population Health and Canadian Community Health Surveys. BMC Women's Health. 2004 Aug 24;4(Suppl I):S35 .

20. Rao JN. Méthodes bootstrap pour l'analyse de données d'enquête à plan de sondage complexe. La série des symposiums internationaux de Statistique Canada : recueil. Symposium 2006 - Enjeux méthodologiques reliés à la mesure de la santé des populations; 2006. (n` au catalogue 11-522XIF)

21. Gee ME, Campbell NR, Gwadry-Sridhar F et collab.; Outcomes Research Task Force of the Canadian Hypertension Education Program. Antihypertensive medication use, adherence, stops and starts in Canadians with hypertension. Can J Cardiol. 2012 May;28(3):383-9.

22. Agence de la santé publique du Canada. L'hypertension [Internet]. Ottawa (Ont.) : Agence de la santé publique du Canada; [mis à jour le 10 décembre 2009; consulté le 29 mars 2011]. Consultable à la page : http://www.phac-aspc.gc.ca/cd-mc/slcdcfs -epamccfi/hypertension-fra.php

23. Walker RL, Gee ME, Bancej C et collab. Health behaviour advice from health professionals to Canadian adults with hypertension : results from a national survey. Can J Cardiol. 2011 Jul-Aug;27(4):446-54.

24. Gee ME, Bienek A, Campbell NRC et collab. Prevalence of, and barriers to, preventive lifestyle behaviours in hypertension (from a national survey of Canadians with hypertension). Am J Cardiol. 2012 Feb;109(4): 570-5.

25. Bancej CM, Campbell N, McKay DW, Nichol M, Walker R, Kaczorowski J. Home blood pressure monitoring among Canadian adults with hypertension : results from the 2009 Survey on Living with Chronic Diseases in Canada. Can J Cardiol. 2010 May;26(5):e152-7.
26. Gee ME, Campbell NR, Bancej CM et collab. Perception of uncontrolled blood pressure and behaviours to improve blood pressure: findings from the 2009 Survey on Living with Chronic Diseases in Canada. J Hum Hypertens. 2011 Mar; 26(3):188-95; doi:10.1038/jhh.2011.5.

27. Chiu M, Austin PC, Manuel DG, Tu JV. Comparison of cardiovascular risk profiles among ethnic groups using population health surveys between 1996 and 2007. CMAJ. 2010 May;182(8):E301-10.

28. Statistique Canada. Autres périodes de référence - Enquête sur les personnes ayant une maladie chronique au Canada (EPMCC) [Internet]. Ottawa (Ont.) : Statistique Canada; 2011 [consulté le 22 mars 2011]. Consultable à la page : http:// www23.statcan.gc.ca/imdb/p2SV_f.pl? Function $=$ getInstanceList $\&$ SurvId $=38899$ \&SurvVer $=2 \&$ InstaId $=38900 \&$ SDDS $=5160$ \&lang $=\mathrm{en} \& \mathrm{db}=\mathrm{imdb} \& \mathrm{adm}=8 \& \mathrm{dis}=2$

29. Statistique Canada. Enquête sur les personnes ayant des problèmes neurologiques au Canada [Internet]. Ottawa (Ont.) : Statistique Canada; 2012 [consulté le 13 avril 2012]. Consultable à la page : http:// www.statcan.gc.ca/survey-enquete/household-menages/5182-fra.htm 